\title{
Synthesis of Poly(3,4-ethylenedioxythiophene) Microspheres by Ultrasonic Spray Polymerization (USPo)
}

\author{
Yinan Zhang and Kenneth S. Suslick* \\ Department of Chemistry, University of Illinois at Urbana-Champaign, 600 S. Mathews Ave., Urbana, Illinois, 61801, United States.
}

\begin{abstract}
Materials and reagent: 3,4-ethylenedioxythiophene (EDOT), iron(III) p-toluenesulfonate hexahydrate, iron(III) chloride hexahydrate, sodium persulfate, acetonitrile, propylene carbonate, poly (methylmethacrylate) (PMMA, Mw 120,000), and lithium perchlorate were purchased from Sigma Aldrich. All the chemicals were used as received without further purification. Pressure sensitive polypropylene tape (Scotch tape), and copper electrical tape were from 3M Corporation. Carbon paper was purchased from Fuel Cell Store (2050-A).
\end{abstract}

Ultrasonic spray pyrolysis synthesis: PEDOT microspheres were produced using the USP set-up depicted in Figure 1. The operating frequency of the water nebulizer used was 1.7 $\mathrm{MHz}$. The furnace was preheated to the desired temperature (e.g., $150^{\circ} \mathrm{C}$ ). Nitrogen was used as the carrier gas at a flow rate of $1 \mathrm{~L} / \mathrm{min}$. The precursor solution (e.g., $200 \mathrm{mM}$ EDOT and $250 \mathrm{mM} \mathrm{FeCl}_{3}$ ) was prepared and then immediately added to the nebulizing chamber. The dark-blue powders were collected in bubblers containing ethanol and subsequently washed with $50 \%$ ethanol/water and centrifuged for a minimum of 4 times. The final products were dried overnight in a vacuum oven at $100{ }^{\circ} \mathrm{C}$.

Material characterizations: Scanning electron micrographs (SEMs) were taken using a JEOL 7000F field emission (FE)-SEM instrument operated at $10 \mathrm{kV}$. Energy-dispersive Xray spectroscopy (EDX) was available on the 7000F-SEM instrument by use of a Thermo Electron EDX microanalysis system. Transmission electron microscopy (TEM) was conducted using a JEOL 2100 Cryo instrument operated at $200 \mathrm{kV}$. The Brunauer-Emmitt-Teller (BET) specific surface area was measured using a Quantachrome Nova 2200e system. Powder X-ray diffraction (PXRD) patterns were obtained on a Siemens-
Bruker D-5000 XRD instrument operated at $40 \mathrm{kV}$ and $30 \mathrm{~mA}$ ( $\mathrm{Cu} \mathrm{Ka}$ radiation). Elemental analysis was performed using a CE 440 CHN Analyzer and inductively coupled plasma mass spectrometer (ICP-MS) by the Microanalysis Lab of the School of Chemical Sciences. Thermal gravimetric analysis (TGA) was conducted using a Mettler-Toledo TGA/DSC1 LF. Scans were collected in the range of $25^{\circ} \mathrm{C}$ to $660^{\circ} \mathrm{C}$ with a ramping rate of $10^{\circ} \mathrm{C} / \mathrm{min}$ under a dry nitrogen atmosphere.

Electrochemical experiments: Working electrodes were prepared by directly spraying PEDOT microspheres onto preweighed carbon paper. The spray-coated carbon paper was then washed thoroughly with deionized water and ethanol to remove any unreacted precursor species. The PEDOT spray-coated carbon paper was then dried thoroughly under vacuum and weighed to calculate the amount of PEDOT deposited. The cyclic voltammetry was conducted using a $\mathrm{CH}$ Instruments Electrochemical Workstation (potentiostat/galvanostat) with a platinum mesh counter electrode and a silver/silver chloride $(\mathrm{Ag} / \mathrm{AgCl})$ reference electrode. The measurements were made at five different scan rates $(50,100,250,500$, and $1000 \mathrm{mV} / \mathrm{s})$ in $1 \mathrm{M} \mathrm{LiClO}_{4}$ acetonitrile solution. All galvanostatic measurements were performed at a current of $1 \mathrm{~mA} / \mathrm{cm}^{2}$.

PEDOT supercapacitor device fabrication: The electrolyte is made by dissolving $8 \mathrm{~g}$ PMMA in $42 \mathrm{~mL}$ of $1 \mathrm{M} \mathrm{LiClO}_{4}$ acetonitrile/propylene carbonate solution $(5: 2 \mathrm{v} / \mathrm{v})$. The mixture was heated at $100{ }^{\circ} \mathrm{C}$, with vigorous stirring until the solution begins to gel. This electrolyte is then sandwiched between symmetrical PEDOT electrodes. The system is then sealed with polypropylene tape. 
a
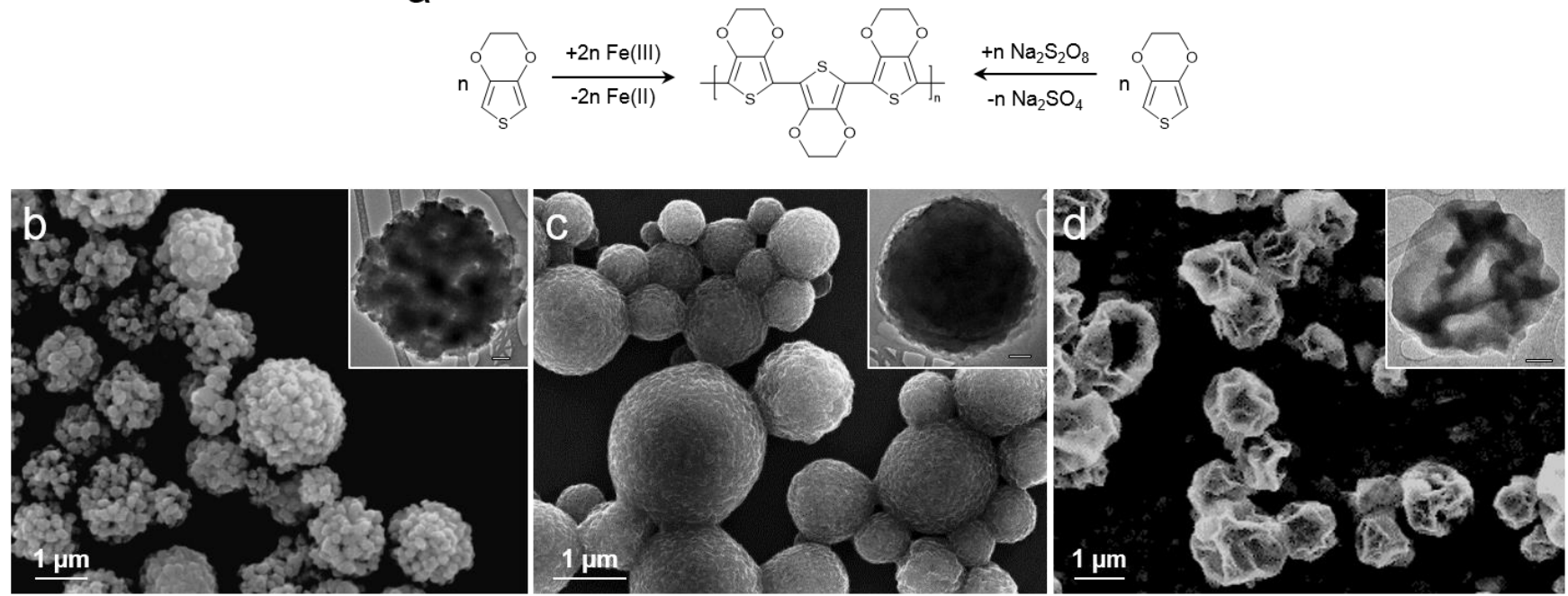

Figure S1. (a) PEDOT synthesis scheme illustrating oxidation with either Fe(III) or persulfate salts. SEM images and TEM images of (b) porous PEDOT microspheres from $\mathrm{FeCl}_{3}$ with diameters of $\approx 1.4 \mu \mathrm{m}$ and BET surface areas of $49 \mathrm{~m}^{2} / \mathrm{g}$, (b) solid PEDOT microspheres from $\mathrm{Fe}(\mathrm{TsO})_{3}$ with diameters of $\approx 1.2 \mu \mathrm{m}$, and $(\mathrm{c})$ hollow PEDOT microspheres from $\mathrm{Na}_{2} \mathrm{~S}_{2} \mathrm{O}_{8}$ with diameters of $\approx 1.9 \mu \mathrm{m}$. The scale bars in the inserts represent $200 \mathrm{~nm}$.

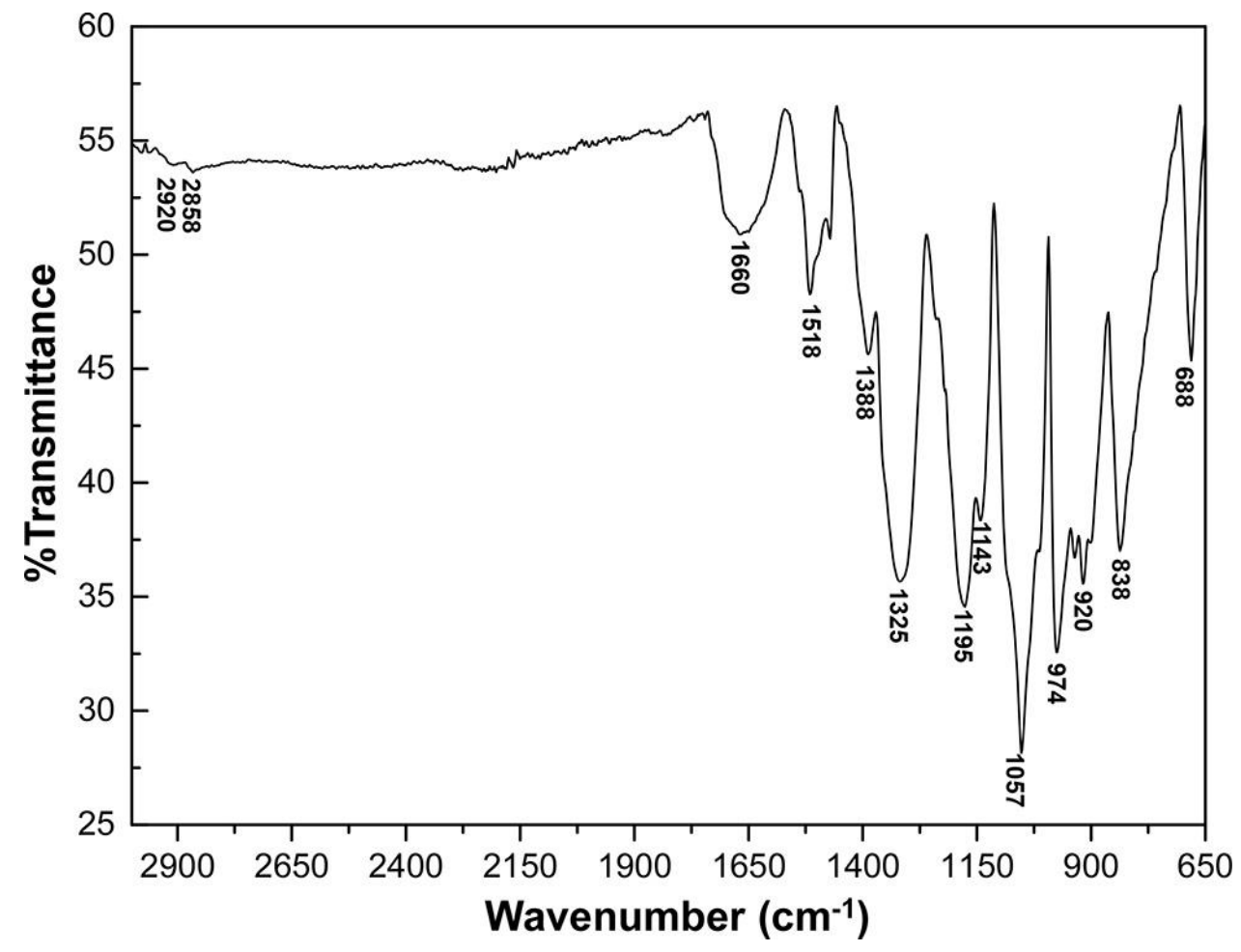

Figure S2. FTIR spectrum of PEDOT microspheres. 


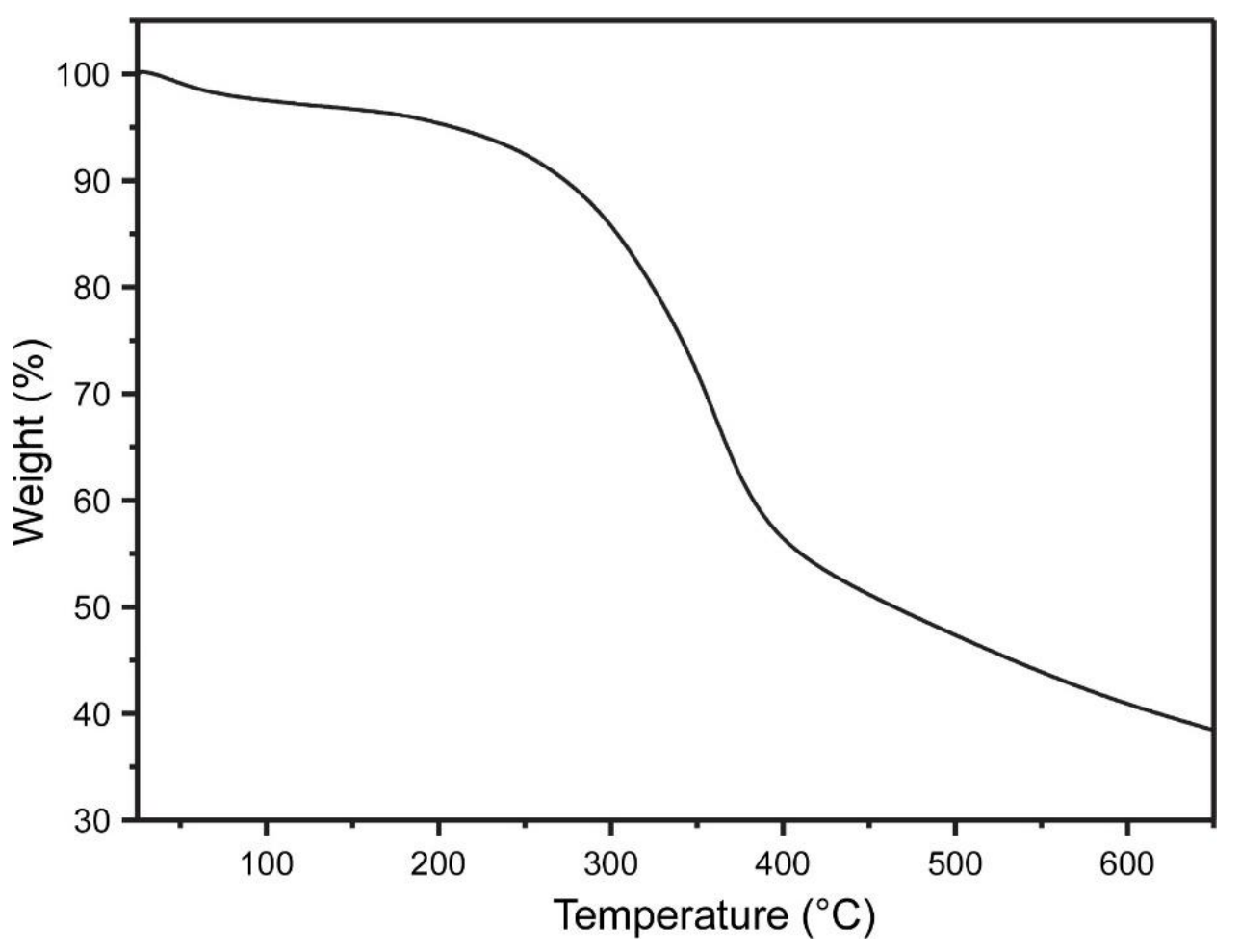

Figure S3. TGA data of PEDOT microspheres showing their thermostability; major decomposition begins its onset around $300{ }^{\circ} \mathrm{C}$.

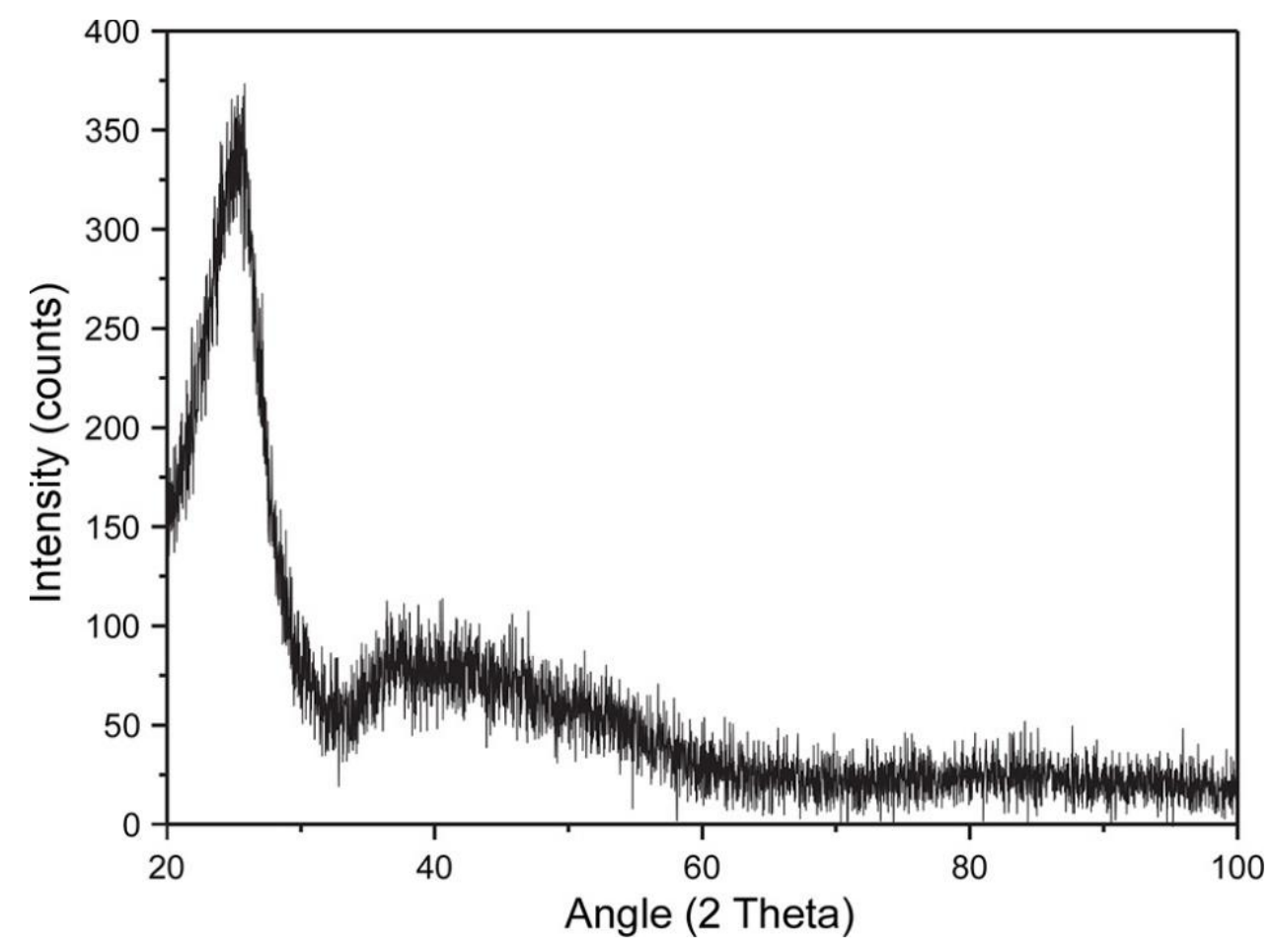

Figure S4. PXRD of PEDOT microspheres. The amorphous polymer gives broad peaks at $\sim 25^{\circ}$ and $\sim 40^{\circ}$. 


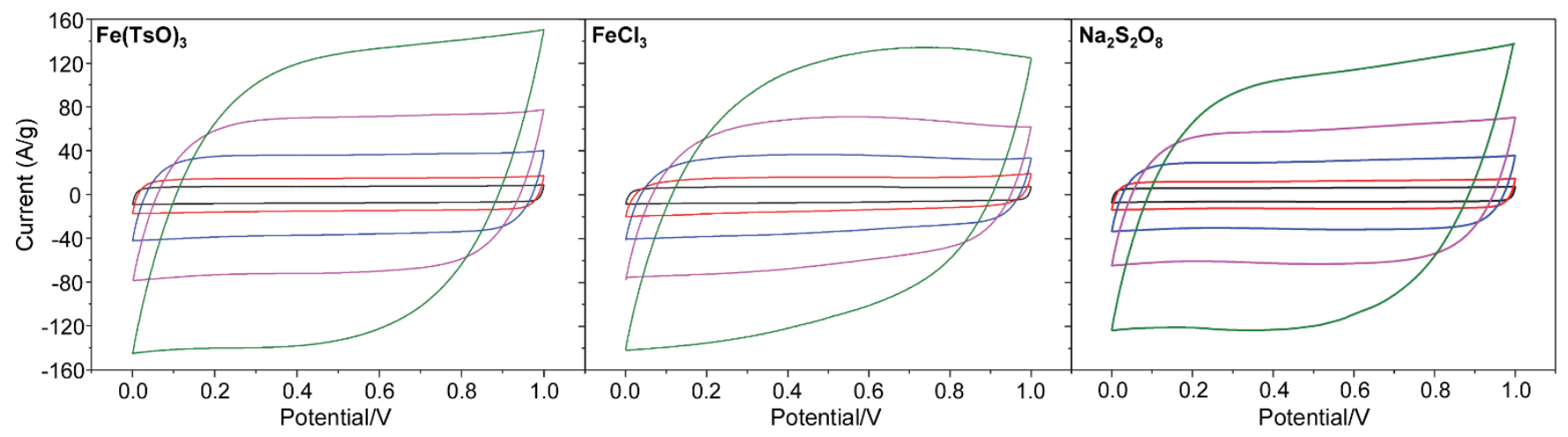

Figure S5. Cyclic voltammogram of PEDOT microspheres synthesized from $\mathrm{Fe}(\mathrm{TsO})_{3}, \mathrm{FeCl}_{3}$, and $\mathrm{Na}_{2} \mathrm{~S}_{2} \mathrm{O}_{8}$ oxidation, as labeled. All products show near ideal charge/discharge cycles for rates as high as $500 \mathrm{mV} / \mathrm{s}$, but with some deviation from ideality at $1000 \mathrm{mV} / \mathrm{s}$.

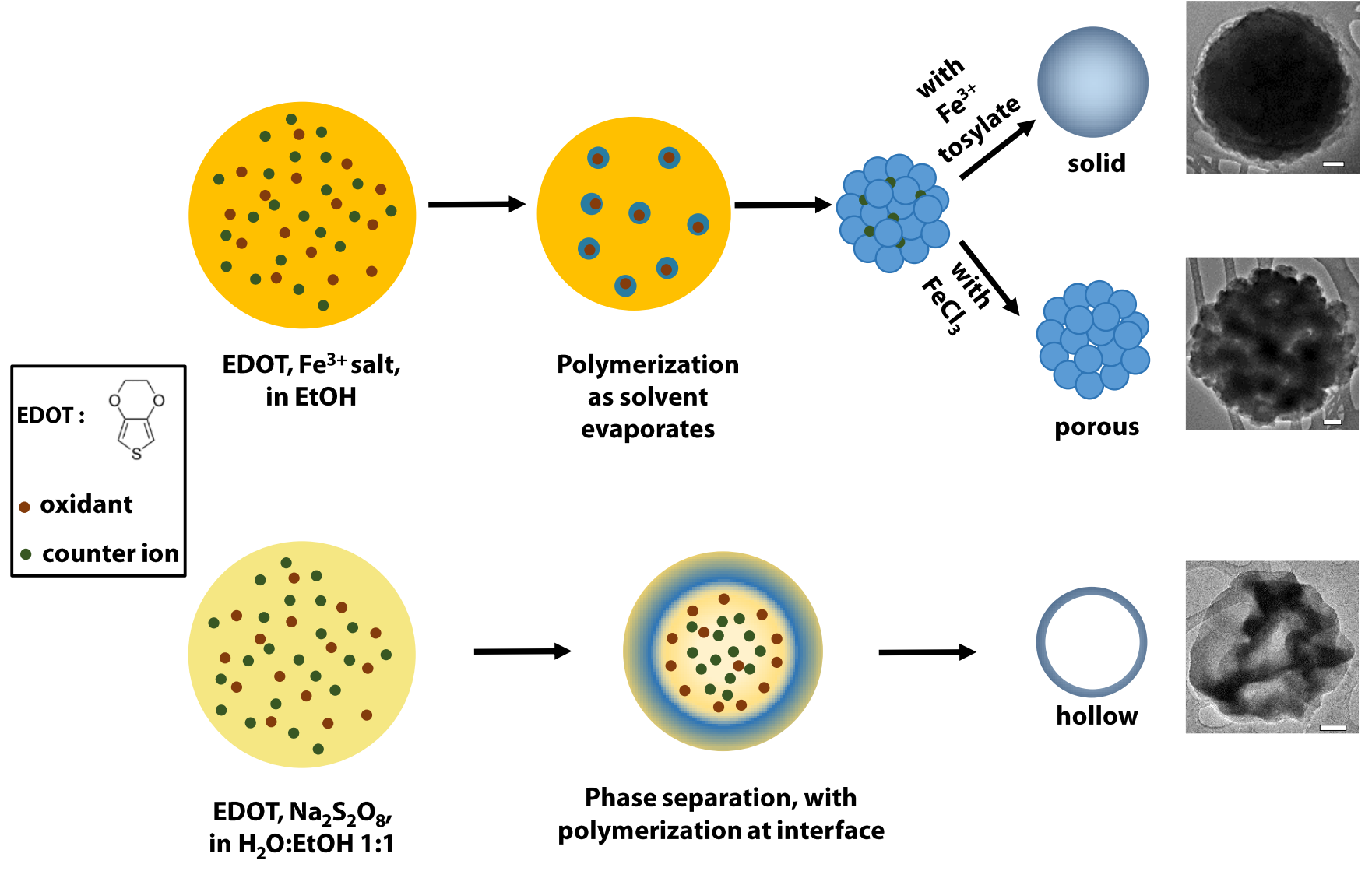

Figure S6. Illustration of hypothetical mechanisms behind the formation of solid, porous, and hollow PEDOT microsphere. Scale bars represent $200 \mathrm{~nm}$. 


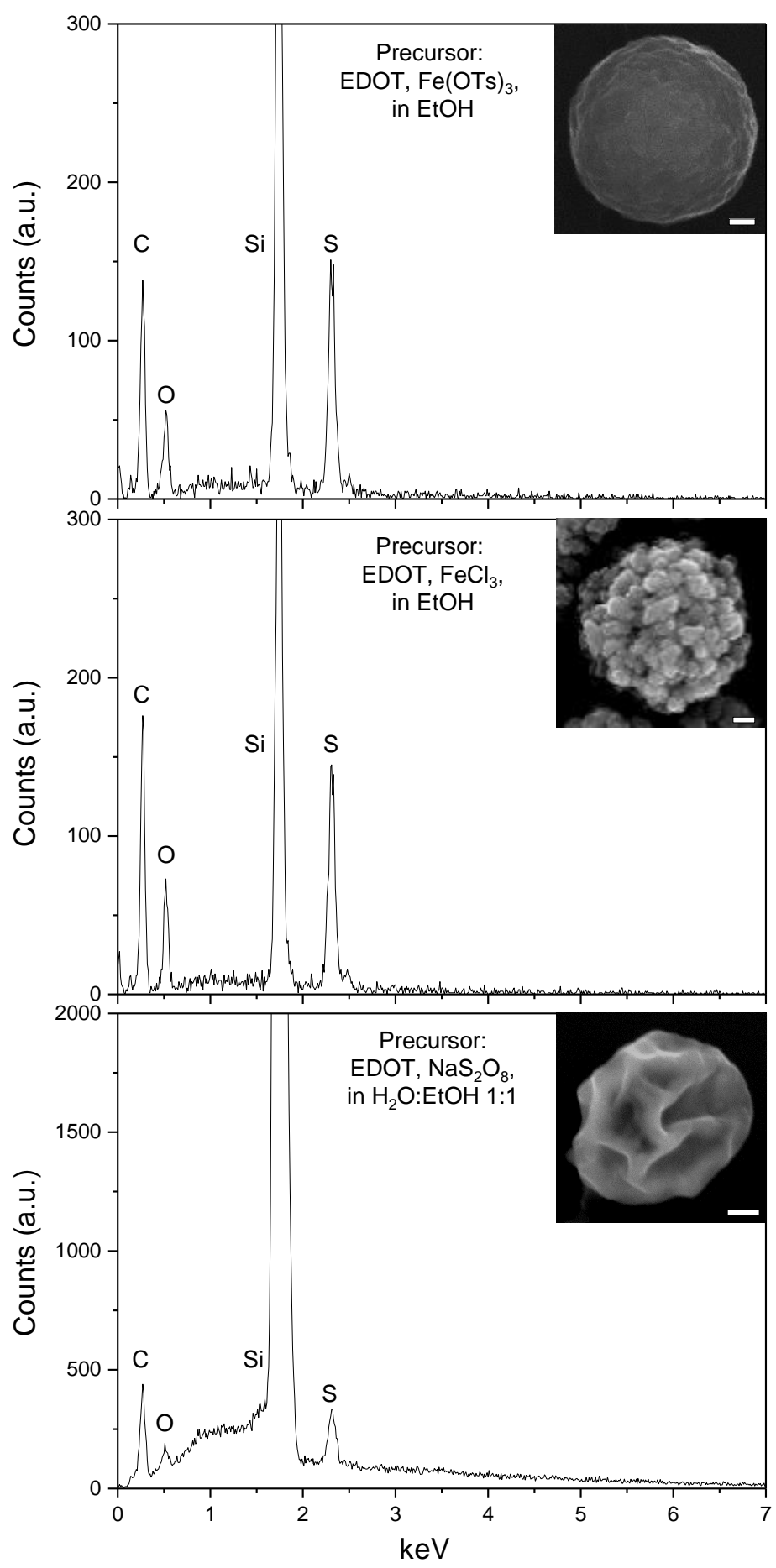

Figure S7. EDX spectra of PEDOT microspheres synthesized from three different conditions. It is clearly that after thorough wash, there is negligible impurities ( such as $\mathrm{Fe}^{3+}$ or $\mathrm{Na}^{+}$) in the final products. Scale bars are each $200 \mathrm{~nm}$. 


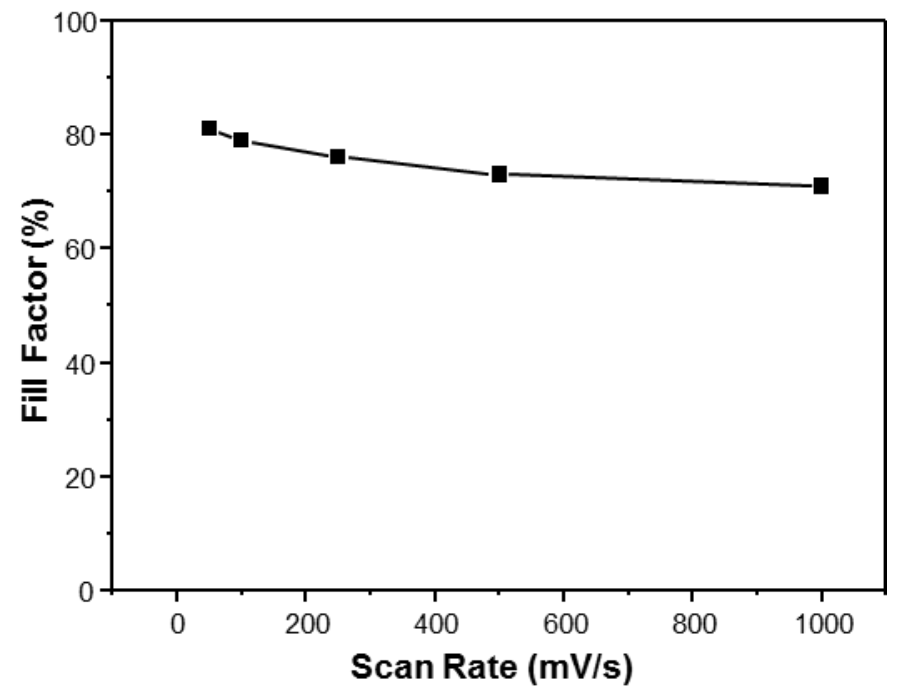

Figure S8. Fill factor calculated from Figure 4(a). The highest fill factor is $81 \%$ at $50 \mathrm{mV} / \mathrm{s}$ and lowest is $71 \%$ at $1000 \mathrm{mV} / \mathrm{s}$.
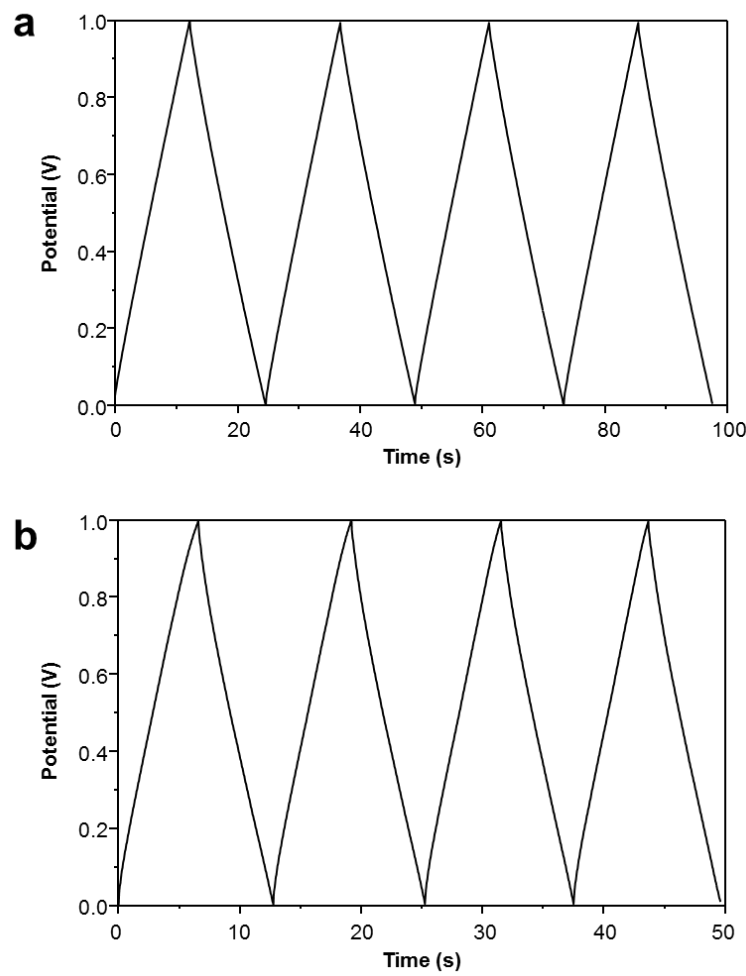

Figure S9. Galvanostatic charge-discharge curves (GV) showing excellent reversibility. (a) GV of three electrode system with PEDOT materials as working electrode, with the reference of $\mathrm{Ag} / \mathrm{AgCl}$. (b) $\mathrm{GV}$ of supercapacitor device with symmetric PEDOT electrodes. 